Published in "Discrete Applied Mathematics 262 (2019) 195-202"

which should be cited to refer to this work.

https://doi.org/10.1016/j.dam.2019.02.010

\title{
Proper circular arc graphs as intersection graphs of paths on a grid
}

\author{
Esther Galby ${ }^{\mathrm{a}, *}$, María Pía Mazzoleni ${ }^{\mathrm{b}, \mathrm{c}}$, Bernard Ries ${ }^{\mathrm{a}}$ \\ ${ }^{a}$ University of Fribourg, Department of Informatics, Decision Support \& Operations Research, Fribourg, Switzerland \\ ${ }^{\mathrm{b}}$ Universidad Nacional de La Plata, Facultad de Ciencias Exactas, Departamento de Matemática, La Plata, Argentina \\ ${ }^{\mathrm{c}}$ CONICET, Argentina
}

\section{A B S T R A C T}

In this paper we present a characterization, by an infinite family of minimal forbidden induced subgraphs, of proper circular arc graphs which are intersection graphs of paths on a grid, where each path has at most one bend (turn).

\section{Introduction}

Intersection graphs of geometrical objects in the plane are among the most studied graph classes and have applications in various domains such as for instance biology, statistics, psychology and computing (see [14]). We define the intersection graph $G$ of a family $\mathcal{F}$ of non empty sets as the graph whose vertices correspond to the elements of $\mathcal{F}$, and two vertices are adjacent in $G$ if and only if the corresponding elements in $\mathcal{F}$ have a non-empty intersection.

Golumbic et al. introduced in [8] the class of edge intersection graphs of paths on a grid (EPG graphs), i.e. graphs for which there exists a collection of nontrivial paths on a rectangular grid in one-to-one correspondence with their vertex set, such that two vertices are adjacent if and only if the corresponding paths share at least one edge of the grid, and showed that every graph is in fact an EPG graph. A natural restriction which was thereupon considered, suggests to limit the number of bends (i.e. $90^{\circ}$ turns at a grid-point) that a path may have; for $k \geq 0$, the class $B_{k}$-EPG consists of those EPG graphs admitting a representation in which each path has at most $k$ bends.

Since their introduction, $B_{k}$-EPG graphs have been extensively studied from several points of view (see for instance $[1-3,5-10,15,16])$. One major interest is the so-called bend number; for a graph class $\mathcal{G}$, the bend number of $\mathcal{G}$ is the minimum integer $k \geq 0$ such that every graph $G \in \mathcal{G}$ is a $B_{k}$-EPG graph. The problem of determining the bend number of graph classes has been widely investigated (see for instance $[3,7,8,10]$ for planar graphs, Halin graphs, line graphs, outerplanar graphs).

Since $B_{0}$-EPG graphs are equivalent to the well-studied class of interval graphs, a particular attention has been paid to $B_{1}$-EPG graphs. The authors in [9] showed that recognizing $B_{1}$-EPG graphs is an NP-complete problem, a result which was further extended to $B_{2}$-EPG graphs in [15]. Therefore, special graph classes were considered. For instance, the authors in [2] provided characterizations of some subclasses of chordal graphs which are $B_{1}$-EPG by families of minimal forbidden induced

\footnotetext{
* Corresponding author.

E-mail addresses: esther.galby@unifr.ch (E. Galby), pia@mate.unlp.edu.ar (M.P. Mazzoleni), bernard.ries@unifr.ch (B. Ries).
} 
subgraphs; in [6], the authors presented a characterization of cographs that are $B_{1}$-EPG and provided a linear time recognition algorithm.

In this paper, we are interested in a subclass of circular arc graphs (CA for short), namely proper circular arc graphs. In [1], the authors showed that CA graphs are $B_{3}$-EPG and further proved that the class of normal circular arc graphs has bend number 2, a result from which we can easily deduce that the bend number of the class of proper circular arc graphs is 2 (see Section 2). They also considered additional constraints on the EPG representations by demanding that the union of the paths lies on the boundary of a rectangle of the grid (EPR graphs). Similarly to EPG graphs, they defined for $k \geq 0$ the class $B_{k}$-EPR and proved that not all circular arc graphs are $B_{3}-E P R$ (it is easily seen that $C A=B_{4}-E P R=E P R$ ). With the intent of pursuing the work done in [1], we here provide a characterization of proper circular arc graphs that are $B_{1}$-EPG by a family of minimal forbidden induced subgraphs (see Section 3) which is a first step towards characterizing the minimal graphs in $\left(C A \cap B_{2}\right.$-EPG) $\backslash\left(C A \cap B_{1}\right.$-EPG). We conclude Section 3 by noting that a characterization by a family of minimal forbidden induced subgraphs of proper circular arc graphs which are $B_{1}$-EPR easily follows from [1] and [12].

\section{Preliminaries}

Throughout this paper, all considered graphs are connected, finite and simple. For all graph theoretical terms and notations not defined here, we refer the reader to [4].

Let $G=(V, E)$ be an undirected graph with vertex set $V$ and edge set $E$. A clique (resp. independent set) is a subset of vertices that are pairwise adjacent (resp. nonadjacent). If $X_{1}$ and $X_{2}$ are two disjoint subsets of vertices, we say that $X_{1}$ is complete to (resp. is anti-complete to) $X_{2}$, which we denote by $X_{1}-X_{2}$ (resp. $X_{1} \cdots X_{2}$ ), if every vertex in $X_{1}$ is adjacent (resp. nonadjacent) to every vertex in $X_{2}$. A dominating set $D$ in $G$ is a subset of vertices such that every vertex not in $D$ is adjacent to at least one vertex in $D$.

We denote by $C_{n}, n \geq 3$, the chordless cycle on $n$ vertices and by $K_{n}, n \geq 1$, the complete graph on $n$ vertices. A $k$-wheel, $k \geq 3$, denoted by $W_{k}$, is a chordless cycle on $k$ vertices with an additional vertex, referred to as the center of the wheel, adjacent to every vertex of the cycle. The 3-sun, denoted by $S_{3}$, consists of an independent set $S=\left\{s_{0}, s_{1}, s_{2}\right\}$ and a clique $K=\left\{k_{0}, k_{1}, k_{2}\right\}$ such that $s_{i}$ is adjacent to $k_{i}$ and $k_{i+1}, i=0,1,2$, where indices are taken modulo 3. Given a graph $G$ and an integer $k \geq 0$, the power graph $G^{k}$ of $G$ has the same vertex set as $G$ with two vertices being adjacent in $G^{k}$ if and only if their distance (i.e. the length of a shortest path between the two vertices) in $G$ is at most $k$.

If $G=(V, E)$ is a graph and $X \subseteq V$ is a subset of vertices, we denote by $G \backslash X$ the graph obtained from $G$ by deleting all vertices in $X$. Equivalently, $G \backslash X$ is the subgraph of $G$ induced by $V \backslash X$, denoted by $G[V \backslash X]$. If $X$ consists of a single vertex, say $X=\{x\}$, we simply write $G \backslash x$. The complement graph of $G$ is the graph $\bar{G}$ having the same vertex set as $G$ with two vertices being adjacent in $\bar{G}$ if and only if they are nonadjacent in $G$. The disjoint union of $G$ and $H$ is denoted by $G \cup H$.

Let $\mathcal{H}$ be a collection of graphs. For $H \in \mathcal{H}$, we say that $G$ contains no induced $H$ if $G$ contains no induced subgraph isomorphic to $H$. A graph is $\mathcal{H}$-free if it contains no induced subgraph isomorphic to some graph belonging to $\mathcal{H}$.

Recall that an interval graph is an intersection graph of intervals on the real line. A graph is said to be chordal if it does not contain any chordless cycle of length at least four as an induced subgraph. An independent set of three vertices such that each pair is joined by a path that avoids the neighborhood of the third is called an asteroidal triple. The following is a well-known characterization of interval graphs.

Theorem 1 ([11]). A graph is an interval graph if and only if it is chordal and contains no asteroidal triple.

A circular arc graph (CA graph) is an intersection graph of open arcs on a circle, i.e. a graph $G=(V, E)$ is a circular arc graph if one can associate an open arc on a circle with each vertex such that two vertices are adjacent if and only if their corresponding arcs intersect. If $\mathcal{C}$ denotes the corresponding circle and $\mathcal{A}$ the corresponding set of arcs, then $\mathcal{R}=(\mathcal{C}, \mathcal{A})$ is called a circular arc representation of $G$. A circular arc graph having a circular arc representation where no two arcs cover the circle is called a normal circular arc graph (NCA graph). A circular arc graph having a circular arc representation where no arc properly contains another is called a proper circular arc graph (PCA graph). It is well known that every PCA graph admits a representation which is simultaneously proper and normal (see [17]); in particular, every PCA graph is a NCA graph. The following theorem provides a minimal forbidden induced subgraph characterization for PCA graphs (see Fig. 1).

Theorem 2 ([18]). A graph is a PCA graph if and only if it is $\left\{G_{i}, C_{n+4} \cup K_{1}, \overline{C_{2 n+3} \cup K_{1}}, \overline{C_{2 n+6}}, 1 \leq i \leq 6, n \geq 0\right\}$-free.

Note that $\overline{C_{2 n+3} \cup K_{1}}$ corresponds to a claw when $n=0$, and to a 5-wheel when $n=1$.

A graph $G$ is a Helly circular arc graph (HCA graph) if it has a circular arc representation in which any subset of pairwise intersecting arcs has a common point on the circle. A graph that admits a circular arc representation which is simultaneously normal and Helly, i.e. no three arcs or less cover the circle, is called a normal Helly circular arc graph (NHCA graph). Similarly, one can define the class of proper Helly circular arc graphs (PHCA graphs) corresponding to those graphs that admit a circular arc representation in which no three arcs cover the circle and no arc properly contains another. It was shown in [13] that a PCA graph is PHCA if it admits a proper circular arc representation in which no two or three arcs cover the circle; in particular, every PHCA graph is a NHCA graph.

Consider a rectangular grid $\mathcal{G}$ where the horizontal lines are referred to as rows and the vertical lines as columns. A gridpoint lying on row $x$ and column $y$ is referred to as $(x, y)$. If $\mathcal{P}$ is a collection of nontrivial simple paths on the grid, the edge 


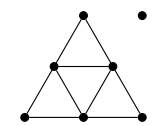

$G_{1}$

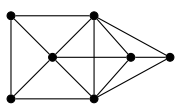

$G_{4}$

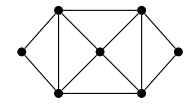

$G_{2}$

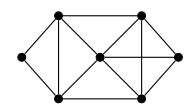

$G_{5}$

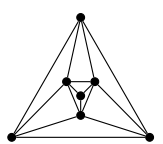

$G_{3}$

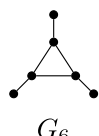

$G_{6}$

Fig. 1. Minimal forbidden induced subgraphs for PCA graphs.

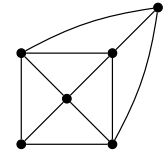

$H_{1}$

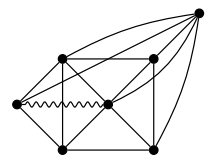

$H_{5}$

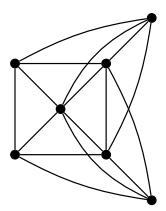

$\mathrm{H}_{2}$

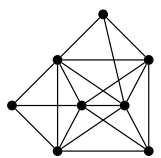

$H_{6}$

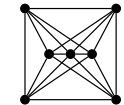

$\mathrm{H}_{3}$

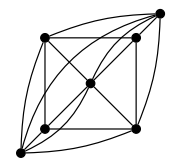

$\mathrm{H}_{7}$

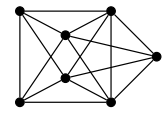

$H_{4}$

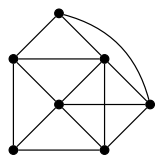

$\mathrm{H}_{8}$

Fig. 2. PCA graphs which are minimally non $B_{1}$-EPG (the serpentine line connecting two vertices indicates the existence of either an edge or a nonedge between those two vertices).

intersection graph $G$ of $\mathcal{P}$ is the graph whose vertex set is in one-to-one correspondence with $\mathcal{P}$ and two vertices are adjacent if and only if the corresponding paths share at least one grid-edge. The path representing some vertex $v$ will be denoted by $P_{v}$. Then $(\mathcal{G}, \mathcal{P})$ is referred to as an EPG representation of $G$ or a $k$-bend EPG representation of $G$ if every path of $\mathcal{P}$ has at most $k$-bends (i.e. $90^{\circ}$ turns at a grid-point) with $k \geq 0$. The class of graphs admitting a $k$-bend EPG representation is called $B_{k}-E P G$. The bend number of a graph $G$ is the smallest $k \geq 0$ such that $G$ is $B_{k}-E P G$; the bend number of a graph class $\mathcal{G}$ is the maximum bend number among all graphs in $\mathcal{G}$.

A graph $G$ is said to be an edge intersection graph of paths on a rectangle (EPR graph) if there exists a set of paths $\mathcal{P}$ on a rectangle $\mathcal{R}$ of the grid in one-to-one correspondence with the vertex set of $G$, where two vertices are adjacent in $G$ if and only if their corresponding paths share at least one grid-edge; $(\mathcal{G}, \mathcal{R}, \mathcal{P})$ is then referred to as an EPR representation of $G$. For $k \geq 0$, we denote by $B_{k}$-EPR the class of graphs for which there exists an EPR representation where every path has at most $k$ bends.

The authors in [1] proved that NCA graphs have a bend number of at most 2 and presented an infinite family of NCA graphs, namely $\left\{C_{4 k-1}^{k}, k \geq 2\right\}$, which are not $B_{1}$-EPG. Since any $C_{4 k-1}^{k}, k \geq 2$ is in fact a PCA graph, we deduce the following corollary from the fact that PCA $\subset$ NCA.

Corollary 3. The class of PCA graphs has bend number 2.

\section{Proper circular arc $B_{1}$-EPG graphs}

As we have seen in Section 2, the bend number of proper circular arc graphs is at most 2 . In this section, we provide a characterization, by a family of minimal forbidden induced subgraphs (see Fig. 2), for PCA graphs which are $B_{1}$-EPG.

Theorem 4. Let $G$ be a PCA graph. Then $G$ is $B_{1}$-EPG if and only if $G$ is $\left\{H_{i}, C_{4 k-1}^{k}, 1 \leq i \leq 8, k \geq 2\right\}$-free.

Proof. Necessary condition. Let us show that for all $1 \leq i \leq 8, H_{i}$ is not $B_{1}$-EPG. Observe that all these graphs contain an induced 4-wheel; we denote by $a, b, c$ and $d$ the four vertices of the 4-cycle of the 4-wheel, and $e$ (one of) its center(s). As shown in [2], this 4-cycle can only be represented by either a true pie or a false pie as $\mathcal{P}_{e}$ should intersect all four corresponding paths of the cycle (see Figs. $3 a$ and $4 a$ ). 


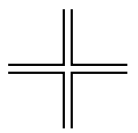

(a) A true pie.

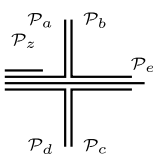

(b) Vertex $z$ is adjacent to $a, d$ and a center (left) or not (right).

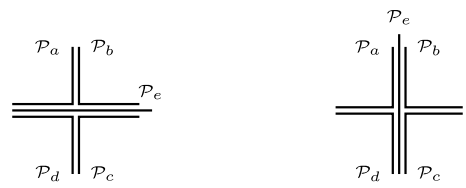

(c) Representations of $W_{4}$ with a true pie.
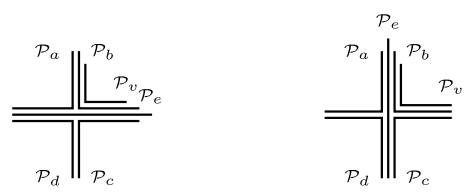

(d) Vertex $v$ is adjacent to $a, b$ and $c$.

Fig. 3. $B_{1}$-EPG representations using a true pie.

Assume first that the 4-wheel is represented by a true pie using column $x$ and row $y$ of the grid. Then, $\mathcal{P}_{e}$ lies either on column $x$ or row $y$ and strictly contains the grid-point $(x, y)$, since it must intersect every path of the 4-cycle (see Fig. $3 c$ ). Consequently, $\mathrm{H}_{3}$ cannot be represented using a true pie.

If a vertex $v$ is adjacent to three vertices of the 4-cycle, say $a, b$ and $c$, its corresponding path must lie on row $x$ and/or column $y$, since it has to intersect $\mathcal{P}_{a}, \mathcal{P}_{b}$ and $\mathcal{P}_{c}$. However, $\mathcal{P}_{v}$ cannot lie entirely on column $x$ or row $y$ as it would otherwise intersect $\mathcal{P}_{d}$. Hence, it lies on both similarly to $\mathcal{P}_{b}$; but then $\mathcal{P}_{v}$ necessarily intersects $\mathcal{P}_{e}$, which implies that $H_{1}$ cannot be represented using a true pie (see Fig. 3d).

If a second vertex $w$ intersects three different vertices of the 4-cycle, we distinguish two cases. Either those vertices are $b$, $c$ and $d$ (the case where they are $d, a$ and $b$ is symmetric), then as shown previously, $\mathcal{P}_{w}$ lies on column $x$ and row $y$ similarly to $\mathcal{P}_{c}$, and therefore intersects $\mathcal{P}_{v}$. Or they are $c, d$ and $a$ in which case $\mathcal{P}_{w}$ lies on column $x$ and row $y$ similarly to $\mathcal{P}_{d}$, and consequently shares only the grid-point $(x, y)$ with $\mathcal{P}_{v}$. Hence, neither $\mathrm{H}_{2}$ nor $\mathrm{H}_{7}$ can be represented using a true pie.

If $z$ is a vertex adjacent to exactly two consecutive vertices of the 4-cycle, say $a$ and $d$, then $\mathcal{P}_{z}$ uses row $y$, where $\mathcal{P}_{a}$ and $\mathcal{P}_{d}$ intersect, without strictly containing the grid-point $(x, y)$ as otherwise it would intersect other vertices of the 4-cycle (note that $\mathcal{P}_{z}$ may then only intersect centers of the 4-wheel which are adjacent and lie on row $y$ ). Hence, $H_{4}$ and $H_{5}$ cannot be represented using a true pie (see Fig. $3 \mathrm{~b}$ ).

Finally, since a vertex $t$ adjacent to only $a$ and $b$ would use column $x$, where $\mathcal{P}_{a}$ and $\mathcal{P}_{b}$ intersect, without strictly containing the grid-point $(x, y), H_{6}$ and $H_{8}$ cannot be represented using a true pie.

Second, assume that the 4-wheel is represented by a false pie using column $x$ and row $y$ of the grid. Then, $\mathcal{P}_{e}$ lies on column $x$ and row $y$ and strictly contains the grid-point $(x, y)$ in order to intersect every path of the 4-cycle (see for instance Fig. 4c). This implies in particular that $\mathrm{H}_{3}$ cannot be represented using a false pie.

If a vertex $v$ is adjacent to three vertices of the 4-cycle, say $a, b$ and $c$, then the path $\mathcal{P}_{v}$ lies on the grid similarly to $\mathcal{P}_{b}$ in order to intersect all three paths without intersecting $\mathcal{P}_{d}$. But then, $\mathcal{P}_{v}$ would intersect $\mathcal{P}_{e}$ which implies that $H_{1}$ cannot be represented using a false pie (see Fig. 4b).

If a second vertex $w$ intersects three different vertices of the 4-cycle, we distinguish two cases. Either those vertices are $b, c$ and $d$ (the case where they are $d, a$ and $b$ is symmetric), then $\mathcal{P}_{w}$ lies on the grid similarly to $\mathcal{P}_{c}$, and therefore intersects $\mathcal{P}_{v}$. Or they are $c, d$ and $a$ in which case $\mathcal{P}_{w}$ lies on the grid similarly to $\mathcal{P}_{d}$, and consequently shares only the grid-point $(x, y)$ with $\mathcal{P}_{v}$. Hence, neither $\mathrm{H}_{2}$ nor $\mathrm{H}_{7}$ can be represented using a false pie.

If $z$ is a vertex adjacent to exactly two consecutive vertices of the 4-cycle, say $a$ and $d$, then $\mathcal{P}_{z}$ uses the grid line where $\mathcal{P}_{a}$ and $\mathcal{P}_{d}$ intersect, without strictly containing the grid-point $(x, y)$ as otherwise it would intersect other vertices of the 4-cycle (note that $\mathcal{P}_{z}$ may then only intersect centers of the 4 -wheel which are adjacent and use the same line as $\mathcal{P}_{z}$ ). Hence, $H_{4}$ and $\mathrm{H}_{5}$ cannot be represented using a false pie (see Fig. 4d).

Finally, since a vertex $t$ adjacent to only $a$ and $b$ would use the line where $\mathcal{P}_{a}$ and $\mathcal{P}_{b}$ intersect, without strictly containing the grid-point $(x, y), H_{6}$ and $H_{8}$ cannot be represented using a false pie.

It follows that no $H_{i}(1 \leq i \leq 8)$ is $B_{1}$-EPG. We conclude this part of the proof by noticing that $C_{4 k-1}^{k} \notin B_{1}$-EPG for $k \geq 2$, as shown in [1].

Sufficient condition. Let $G=(V, E)$ be a PCA graph which is $\left\{H_{i}, C_{4 k-1}^{k}, 1 \leq i \leq 8, k \geq 2\right\}$-free. Consider a normal proper representation $\mathcal{R}=(\mathcal{C}, \mathcal{A})$ of $G$, where $\mathcal{C}$ is a circle and $\mathcal{A}$ is a set of open arcs of $\mathcal{C}$ in one-to-one correspondence with the vertices of $G$ (notice that such a representation exists due to [17]). Before turning to the proof, let us first make the following observation.

Observation 1. Any set of arcs in $\mathcal{A}$ covering the circle $\mathcal{C}$ corresponds to a dominating set in $G$. In particular, if $G$ contains a 4-wheel as an induced subgraph, then $G$ has a dominating triangle. 


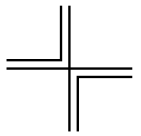

(a) A false pie.
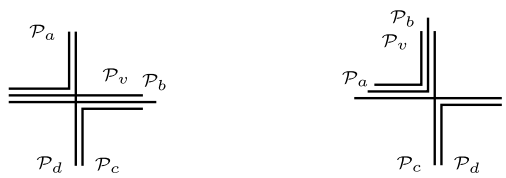

(b) Vertex $v$ is adjacent to $a, b$ and $c$.
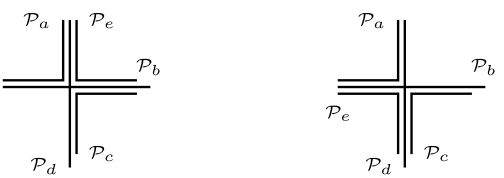

(c) Representations of $W_{4}$ with a false pie.
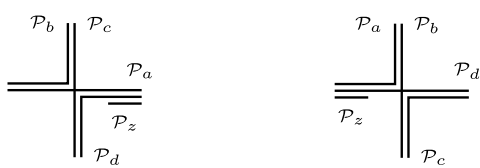

(d) Vertex $z$ is adjacent to $a$ and $d$.

Fig. 4. $B_{1}$-EPG representations using a false pie.

Proof. It is clear that such a set of arcs corresponds to a dominating set in $G$. If $G$ contains a 4-wheel, then the arcs corresponding to 4 -cycle $C$ of the 4 -wheel cover the circle $\mathcal{C}$. But then the arc representing the center of the 4-wheel together with two arcs corresponding to two vertices of $C$ must also cover $\mathcal{C}$, i.e. $G$ has a dominating triangle. $\diamond$

If we assume that no three $\operatorname{arcs}$ in $\mathcal{A} \operatorname{cover} \mathcal{C}$, then $G$ is PHCA and the result follows from the fact that PHCA $\left\{C_{4 k-1}^{k}\right\}_{k \geq 2}$-free $\subset \mathrm{NHCA} \cap\left\{C_{4 k-1}^{k}\right\}_{k \geq 2}$-free $=B_{1}$-EPR (see [1]). Hence, we may assume that there exist three arcs in $\mathcal{A}$ covering $\mathcal{C}$. If $G$ contains a 4-wheel, let $C=\left\{x_{1}, x_{2}, x_{3}\right\}$ denote the dominating triangle following from Observation 1 , with $x_{1}$ being the center of the 4-wheel. Otherwise, let $C=\left\{x_{1}, x_{2}, x_{3}\right\}$ be any triangle whose corresponding arcs cover $\mathcal{C}$. In both cases, each vertex is adjacent to at least two vertices of $C$ as $\mathcal{R}$ is a proper representation. For $j \in\{1,2,3\}$, denote by $\mathcal{A}_{j, j+1}=\left\{x \in V \mid x x_{j-1} \notin E\right\}$, where indices are taken modulo 3 , the subset of vertices adjacent to only $x_{j}$ and $x_{j+1}$. Note that each $\mathcal{A}_{j, j+1}$ is a clique as $G$ would otherwise contain an induced claw, namely $x_{j+1}, x_{j-1}, x, x^{\prime}$ for any two $x, x^{\prime} \in \mathcal{A}_{j, j+1}$ such that $x x^{\prime} \notin E$. Similarly, consider the subset of vertices $\mathcal{A}_{c}=\left\{x \in V \mid \forall j \in\{1,2,3\}, x x_{j} \in E\right\}$ adjacent to all three vertices of $C$. We now distinguish cases depending on whether $G$ contains a 4-wheel as an induced subgraph or not.

Case 1 ( $G$ Contains an Induced 4-wheel). According to the above, $\mathcal{A}_{1,2}$ and $\mathcal{A}_{1,3}$ are not anticomplete. Thus, there exist $x_{4} \in \mathcal{A}_{1,3}$ and $x_{5} \in \mathcal{A}_{1,2}$ such that $x_{4} x_{5} \in E$, which together with $x_{2}$ and $x_{3}$ form the 4-cycle $C^{\prime}$ of the 4-wheel. Since $C^{\prime}$ is dominating and $G$ has no induced claw, each remaining vertex of $G$ is adjacent to at least two vertices of $C^{\prime}$. Consider accordingly the subset of vertices $\mathcal{A}_{3,4}$ (resp. $\mathcal{A}_{4,5}, \mathcal{A}_{5,2}$ ) adjacent to only $x_{3}$ and $x_{4}$ (resp. $x_{4}$ and $x_{5}, x_{5}$ and $x_{2}$ ), the subset of vertices $\mathcal{A}_{2}$ (resp. $\mathcal{A}_{3} ; \mathcal{A}_{4} ; \mathcal{A}_{5}$ ) adjacent to only $x_{5}, x_{2}$ and $x_{3}$ (resp. $x_{2}, x_{3}$ and $x_{4} ; x_{3}, x_{4}$ and $x_{5} ; x_{4}, x_{5}$ and $x_{2}$ ) and the subset of vertices $\mathcal{A}_{\mathcal{C}^{\prime}}=\left\{x \in V \mid \forall i \in\{2,3,4,5\}, x x_{i} \in E\right\}$ adjacent to all vertices of $C^{\prime}$ (note that $x_{1} \in \mathcal{A}_{\mathcal{C}^{\prime}}$ ). Since $G$ contains no induced claw, each $\mathcal{A}_{j, j+1}$ is a clique, as well as each $\mathcal{A}_{j}$. Furthermore, since $G$ contains no induced:

- $H_{1}$, each $\mathcal{A}_{j}$ is complete to $\mathcal{A}_{c^{\prime}}$, for $j=2,3,4,5$;

- $H_{2}$, we have $\mathcal{A}_{2}-\mathcal{A}_{3}-\mathcal{A}_{4}-\mathcal{A}_{5}-\mathcal{A}_{2}$;

- $H_{7}$, we have $\mathcal{A}_{2} \cdots \mathcal{A}_{4}$ and $\mathcal{A}_{3} \cdots \mathcal{A}_{5}$;

- $H_{5}$, we have $\mathcal{A}_{j, k} \cdots \mathcal{A}_{i}$ for all $i \neq j, k$ with $(j, k) \in\{(2,3),(3,4),(4,5),(5,2)\}$;

- $H_{8}$ and 5-wheel (by Theorem 2), we have $\mathcal{A}_{2,3} \cdots \mathcal{A}_{3,4} \cdots \mathcal{A}_{4,5} \cdots \mathcal{A}_{5,2} \cdots \mathcal{A}_{2,3}$;

- $\overline{C_{6}}$ (by Theorem 2), we have $\mathcal{A}_{2,3} \cdots \mathcal{A}_{4,5}$ and $\mathcal{A}_{3,4} \cdots \mathcal{A}_{5,2}$;

- claw (by Theorem 2), we have $\mathcal{A}_{j}-\mathcal{A}_{j, k}-\mathcal{A}_{k}$ for $(j, k) \in\{(2,3),(3,4),(4,5),(5,2)\}$.

Now, if we assume that both $\mathcal{A}_{2,3}$ and $\mathcal{A}_{4,5}$ are nonempty, then both are complete to $\mathcal{A}_{c^{\prime}}$ as $G$ would otherwise contain either $G_{2}$ or $G_{5}$ as an induced subgraph (see Fig. 1). But then $\mathcal{A}_{c^{\prime}}$ is a clique since $G$ does not contain $H_{4}$ as an induced subgraph. Consequently, $\mathcal{A}_{3,4}$ and $\mathcal{A}_{5,2}$ cannot both be nonempty; if it were indeed the case, both $\mathcal{A}_{3,4}$ and $\mathcal{A}_{5,2}$ would also be complete to $\mathcal{A}_{c^{\prime}}$, and $G$ would then contain an induced claw, namely $x_{1}, v, w$ and $z$, with $v \in \mathcal{A}_{2,3}, w \in \mathcal{A}_{3,4}$ and $z \in \mathcal{A}_{4,5}$. Hence, we may assume, without loss of generality, that $\mathcal{A}_{5,2}=\emptyset$ (the same reasoning applies if we assume that $\mathcal{A}_{3,4}=\emptyset$ ). But then $\mathcal{A}_{c^{\prime}}$ and $\mathcal{A}_{3,4}$ must be anti-complete as $G$ would otherwise contain an induced claw (the same as before), and $G$ is consequently $B_{1}$-EPG (see Fig. 5).

Now, assume without loss of generality, that $\mathcal{A}_{2,3}=\mathcal{A}_{5,2}=\emptyset$ and $\mathcal{A}_{3,4}, \mathcal{A}_{4,5} \neq \emptyset$. We know from the above that $\mathcal{A}_{3,4}$ is anti-complete to $\mathcal{A}_{4,5}$. Also, for all $x \in \mathcal{A}_{\mathcal{c}^{\prime}}, x$ must be either complete to $\mathcal{A}_{3,4}$ and anti-complete to $\mathcal{A}_{4,5}$ or, conversely, anti-complete to $\mathcal{A}_{3,4}$ and complete to $\mathcal{A}_{4,5}$, as $G$ would otherwise contain an induced claw; indeed, if there exist $x^{\prime} \in \mathcal{A}_{3,4}$ and $x^{\prime \prime} \in \mathcal{A}_{4,5}$ such that $x$ is nonadjacent (resp. adjacent) to both $x^{\prime}$ and $x^{\prime \prime}$, then $x_{4}, x^{\prime}, x^{\prime \prime}$ and $x$ (resp. $x, x^{\prime}, x^{\prime \prime}$ and $x_{2}$ ) induce a claw. Thus, we can partition $\mathcal{A}_{c^{\prime}}$ into subsets $\mathcal{A}_{c^{\prime}}^{i}=\left\{x \in \mathcal{A}_{c^{\prime}} \mid \forall x^{\prime} \in \mathcal{A}_{i+2, i+3}, x x^{\prime} \in E\right\}$ for $i=1,2$, and, since $G$ is $H_{4}$-free, both of these subsets are cliques. Assuming $\mathcal{A}_{c^{\prime}}^{1}$ and $\mathcal{A}_{c^{\prime}}^{2}$ are non empty, there cannot exist $x \in \mathcal{A}_{c^{\prime}}^{1}$ and $x^{\prime} \in \mathcal{A}_{c^{\prime}}^{2}$ such that $x x^{\prime} \notin E$ since $G$ would otherwise contain an induced $G_{2}$ (see Fig. 1 ); indeed, $x_{3}, x, x_{5}, x^{\prime}$ and $x_{2}$ would form a 4-wheel, with 


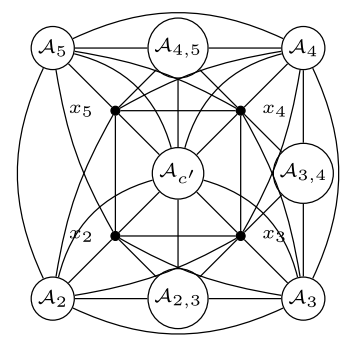

(a) General structure of $G$.

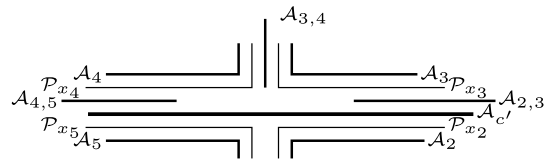

(b) A $B_{1}$-EPG representation of $G$.

Fig. 5. $G$ contains $W_{4}$ and both $\mathcal{A}_{2,3}$ and $\mathcal{A}_{4,5}$ are nonempty.

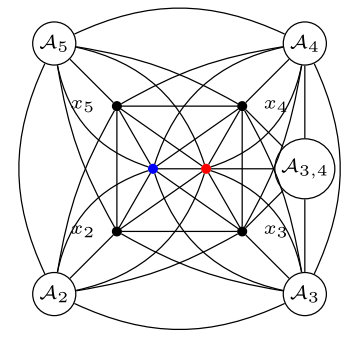

(a) General structure of $G$ (in red: $\mathcal{A}_{c^{\prime}}^{a}$; in blue: $\mathcal{A}_{c^{\prime}}^{\text {na }}$ ).

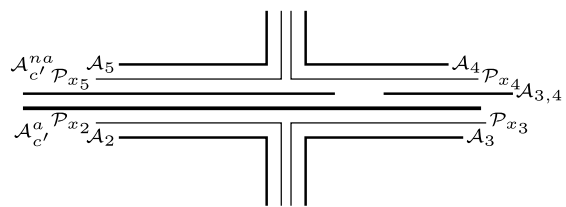

(b) A $B_{1}$-EPG representation of $G$.

Fig. 6. $G$ contains $W_{4}$ and only $\mathcal{A}_{3,4}$ is non empty.
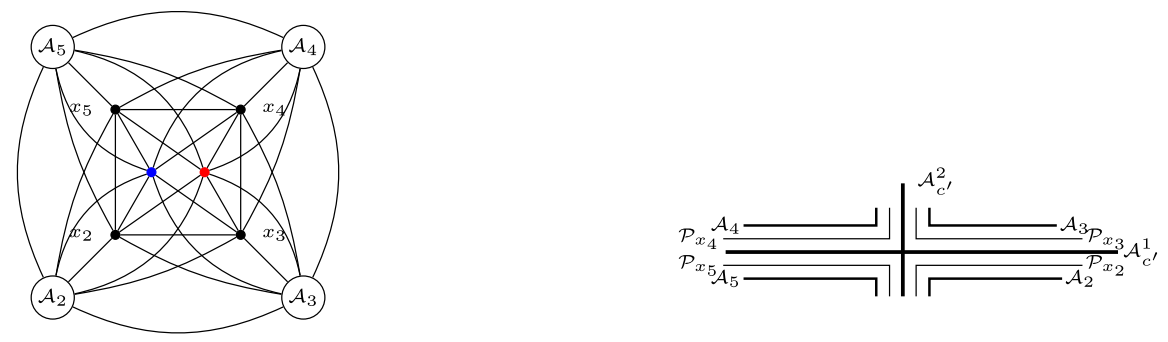

(a) General structure of $G$ (in red: $\mathcal{A}_{c^{\prime}}^{1}$; in blue: $\mathcal{A}_{c^{\prime}}^{2}$ ).

(b) A $B_{1}$-EPG representation of $G$.

Fig. 7. $G$ contains $W_{4}$ and each $\mathcal{A}_{j, j+1}$ is empty.

one vertex of $\mathcal{A}_{3,4}$ adjacent to only $x_{3}$ and $x$, and one vertex of $\mathcal{A}_{4,5}$ adjacent to only $x_{5}$ and $x^{\prime}$. Hence, $\mathcal{A}_{c^{\prime}}^{1} \cup \mathcal{A}_{c^{\prime}}^{2}$ is a clique; but then, $G$ contains $H_{6}$ as an induced subgraph. Thus, we have to assume that exactly one of $\mathcal{A}_{c^{\prime}}^{1}$ and $\mathcal{A}_{c^{\prime}}^{2}$ is empty, and $G$ is then $B_{1}$-EPG as an induced subgraph of the previous case.

Suppose now that only one of the $\mathcal{A}_{j, j+1}$ is non empty, for instance $\mathcal{A}_{3,4}$. If $x \in \mathcal{A}_{c^{\prime}}$ is adjacent to some vertex $x^{\prime} \in \mathcal{A}_{3,4}$, then $x$ is complete to $\mathcal{A}_{3,4}$ as $G$ would otherwise contain an induced $G_{4}$ (see Fig. 1). We can therefore partition $\mathcal{A}_{c^{\prime}}$ into two subsets $\mathcal{A}_{c^{\prime}}^{a}=\left\{x \in \mathcal{A}_{c^{\prime}} \mid \forall x^{\prime} \in \mathcal{A}_{3,4}, x x^{\prime} \in E\right\}$ and $\mathcal{A}_{c^{\prime}}^{n a}=\left\{x \in \mathcal{A}_{c^{\prime}} \mid \forall x^{\prime} \in \mathcal{A}_{3,4}, x x^{\prime} \notin E\right\}$. Since $G$ does not contain an induced $H_{4}, \mathcal{A}_{c^{\prime}}^{a}$ must be a clique; and, since $G$ does not contain an induced claw, $\mathcal{A}_{c^{\prime}}^{\text {na }}$ must also be a clique. If one of $\mathcal{A}_{c^{\prime}}^{a}$ and $\mathcal{A}_{c^{\prime}}^{\text {na is }}$ empty, then $G$ is $B_{1}$-EPG as an induced subgraph of the first case. If both are non empty, then $\mathcal{A}_{c^{\prime}}^{a} \cup \mathcal{A}_{c^{\prime}}^{\text {na }}$ is a clique, as $G$ would otherwise contain an induced $G_{3}$ (see Fig. 1 ), and $G$ is consequently $B_{1}$-EPG (see Fig. 6 ).

Finally, if we assume that every $\mathcal{A}_{j, j+1}$ is empty, since $G$ does not contain $\mathrm{H}_{3}$ or a claw as an induced subgraph, we can partition $\mathcal{A}_{c^{\prime}}$ into two cliques, $\mathcal{A}_{c^{\prime}}^{1}$ and $\mathcal{A}_{c^{\prime}}^{2}$, which are anti-complete, and again $G$ is $B_{1}$-EPG (see Fig. 7).

Case 2 ( $G$ Contains No Induced 4-wheel). Assume henceforth that $\mathcal{A}_{1,2}, \mathcal{A}_{2,3}$ and $\mathcal{A}_{3,1}$ are pairwise anti-complete. If all three subsets are non empty, then for all $x \in \mathcal{A}_{c}$, there must exist $j \in\{1,2,3\}$ such that $x$ is complete to both $\mathcal{A}_{j-1, j}$ and $\mathcal{A}_{j, j+1}$, and anti-complete to $\mathcal{A}_{j+1, j+2}$ (otherwise $G$ would contain an induced claw). Hence, we can partition $\mathcal{A}_{c}$ into three subsets $\mathcal{A}_{c}^{j}=\left\{x \in \mathcal{A}_{c} \mid \forall x^{\prime} \in \mathcal{A}_{j-1, j} \cup \mathcal{A}_{j, j+1}, x x^{\prime} \in E\right.$ and $\left.\forall x^{\prime} \in \mathcal{A}_{j+1, j+2}, x x^{\prime} \notin E\right\}(j \in\{1,2,3\})$ which must be cliques since $G$ does not contain an induced claw. But then either $\mathcal{A}_{c}$ is a clique, in which case $G$ is $B_{1}$-EPG (see Fig. 8), or there exists $x \in \mathcal{A}_{c}^{j}$ and 


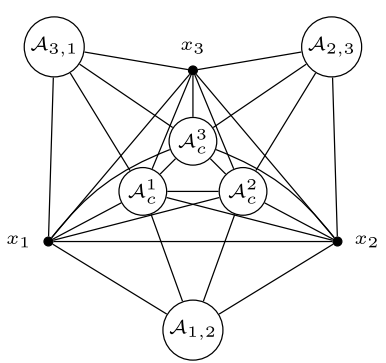

(a) General structure of $G$.

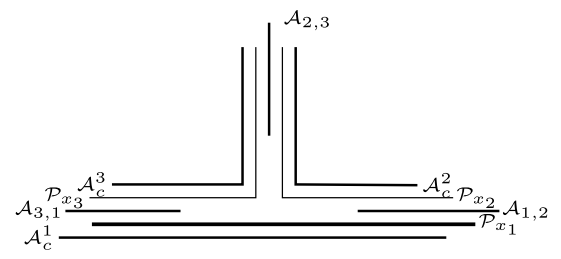

(b) A $B_{1}$-EPG representation of $G$.

Fig. 8. $G$ does not contain $W_{4}$, all three $\mathcal{A}_{j, j+1}$ are nonempty and $\mathcal{A}_{c}$ is a clique.

$x^{\prime} \in \mathcal{A}_{c}^{j+1}$ such that $x x^{\prime} \notin E$, and $G$ contains a 4-wheel with $x, x^{\prime \prime}, x^{\prime}, x_{j+2}$ (for some $x^{\prime \prime} \in \mathcal{A}_{j, j+1}$ ) as its 4-cycle and $x_{j}$ as its center, which is contrary to our assumption.

If we now assume that at least one of the subsets $\mathcal{A}_{j, j+1}$ is empty, then at least one vertex $x_{i}$ of $C$ is adjacent to every vertex of $G$ and $G$ is consequently an interval graph. Indeed, assume that $G \backslash x_{i}$ contains an induced cycle $C^{\prime}=y_{1}, \ldots, y_{l}$ with $l>3$. Then, together with $x_{i}$, it would form an $l$-wheel. But since $G$ is proper, it cannot contain a $k$-wheel with $k>4$. Hence $l=4$ and $G$ would contain a 4 -wheel which is contrary to our assumption. Therefore, $G$ has no induced cycle of length larger than 3, i.e. $G$ is chordal. Furthermore, if there existed three pairwise nonadjacent vertices in $G \backslash x_{i}$, then together with $x_{i}$ they would induce a claw. Hence, $G \backslash x_{i}$ contains no asteroidal triple, i.e. $G$ is an interval graph (see Theorem 1), and therefore $B_{1}$-EPG.

From the characterization of $B_{1}$-EPR graphs, i.e. intersection graphs of paths on a rectangle of a grid where each path has at most one bend, given in [1], we deduce the following characterization by a family of minimal forbidden induced subgraphs of PCA graphs which are $B_{1}$-EPR. It is easily seen that the class of circular arc graphs is exactly the class $B_{4}$-EPR. The authors of [1] further proved that NCA graphs have a bend number, with respect to EPR representations, of at most 2; hence, since PCA $\subset$ NCA, PCA graphs also have a bend number, with respect to EPR representations, of at most 2.

Corollary 5. Let $G$ be a PCA graph. Then $G$ is $B_{1}$-EPR if and only if $G$ is $\left\{W_{4}, S_{3}, C_{4 k-1}^{k}, k \geq 2\right\}$-free.

Proof. It was shown in [1] that for $k \geq 2, C_{4 k-1}^{k} \notin B_{1}$-EPG; a fortiori, $C_{4 k-1}^{k} \notin B_{1}$-EPR. We also know from [2] that $W_{4} \notin B_{1}$-EPR and from [8] that $S_{3} \notin B_{1}$-EPR.

Conversely, it was shown in [12] that PCA $\cap\left\{W_{4}, S_{3}\right\}$-free $=$ PHCA. The result then follows from the fact that PHCA $\cap$ $\left\{C_{4 k-1}^{k}, k \geq 2\right\}$-free $\subset$ NHCA $\cap\left\{C_{4 k-1}^{k}, k \geq 2\right\}$-free $=B_{1}$-EPR (see [1]).

\section{Conclusion}

In this paper, we present characterizations by (infinite) families of minimal forbidden induced subgraphs for $B_{1}$-EPG $\cap$ PCA and $B_{1}$-EPR $\cap$ PCA. This is a first step towards finding a characterization of the minimal graphs in (CA $\cap B_{2}$-EPG) \(CA $\cap B_{1}$-EPG), a question left open in [1].

\section{Acknowledgment}

This research was carried out when Dr M.P. Mazzoleni was visiting the University of Fribourg. The support of this institution is gratefully acknowledged.

\section{References}

[1] L. Alcón, F. Bonomo, G. Durán, M. Gutierrez, M.P. Mazzoleni, B. Ries, M. Valencia-Pabon, On the bend number of circular arc graphs as edge intersection graphs of paths on a grid, Discrete Appl. Math. 234 (2018) 12-21.

[2] A. Asinowski, B. Ries, Some properties of edge intersection graphs of single-bend paths on a grid, Discrete Math. 312 (2) (2012) 427-440.

[3] T. Biedl, M. Stern, Edge-intersection graphs of k-bend paths in grids, in: Computing and Combinatorics: 15th Annual International Conference, COCOON 2009 Niagara Falls, in: 2009 Proceedings, Springer Berlin Heidelberg, NY, USA, Berlin, Heidelberg, 2009, pp. 86-95.

[4] J.A. Bondy, U.S.R. Murty, Graph Theory, Springer, New York, 2007.

[5] K. Cameron, S. Chaplick, C.T. Hoàng, Edge intersection graphs of L-shaped paths in grids, Electron. Notes Discrete Math. 44 (2013) $363-369$.

[6] E. Cohen, M.C. Golumbic, B. Ries, Characterizations of cographs as intersection graphs of paths on a grid, Discrete Appl. Math. 178 (2014) 46-57.

[7] M.C. Francis, A. Lahiri, VPG And EPG bend-numbers of Halin graphs, Discrete Appl. Math. 215 (2016) 95-105.

[8] M.C. Golumbic, M. Lipshteyn, M. Stern, Edge intersection graphs of single bend paths on a grid, Networks 54 (3) (2009) $130-138$.

[9] D. Heldt, K. Knauer, T. Ueckerdt, Edge-intersection graphs of grid paths: The bend-number, Discrete Appl. Math. 167 (2014) 144-162.

[10] D. Heldt, K. Knauer, T. Ueckerdt, On the bend-number of planar and outerplanar graphs, Discrete Appl. Math. 179 (2014) 109-119.

[11] C. Lekkerkerker, J. Boland, Representation of a finite graph by a set of intervals on the real line, Fund. Math. 51 (1) (1962) 45-64. 
[12] M.C. Lin, F.J. Soulignac, J.L. Szwarcfiter, Proper Helly circular-arc graphs, in: Graph-Theoretic Concepts in Computer Science, Springer Berlin Heidelberg, Berlin, Heidelberg, 2007, pp. 248-257.

[13] T.A. McKee, Restricted circular-arc graphs and clique cycles, Discrete Math. 263 (2003) 221-231.

[14] T.A. McKee, F.R. McMorris, Topics in intersection graph theory, in: SIAM Monographs in Discrete Mathematics and Applications, 1999

[15] M. Pergel, P. Rzązewski, On edge intersection graphs of paths with 2 bends, in: Graph-Theoretic Concepts in Computer Science: 42nd International Workshop, WG 2016, in: Revised Selected Papers, Springer Berlin Heidelberg, Istanbul, Turkey, Berlin, Heidelberg, 2016, pp. $207-219$.

[16] B. Ries, Some properties of edge intersection graphs of single-bend paths on a grid, Electron. Notes Discrete Math. 34 (2009) 29-33.

[17] A.C. Tucker, Matrix characterizations of circular arc graphs, Pacific J. Math. 39 (2) (1971) 535-545.

[18] A.C. Tucker, Structure theorems for some circular arc graphs, Discrete Math. 7 (1) (1974) 167-195. 